\title{
Folding of Thin-Walled Composite Structures with a Soft Matrix
}

\author{
Francisco Lopez Jimenez* and Sergio Pellegrino ${ }^{\dagger}$ \\ California Institute of Technology, Pasadena, California 91125
}

\begin{abstract}
The paper presents detailed micromechanical finite element simulations of composite materials with soft materials undergoing large macroscopic bending deformation. The simulations allow the study of fibre microbuckling under bending, including the kinematics of the fibres, as well as the strains in the matrix. These simulations lead to a simple analytical model that allows a quite accurate estimation of the buckling wavelength. It also provides the moment-curvature relationship. Finally, the model is also able to predict the maximum strain in the fibres for a given curvature.
\end{abstract}

\section{Introduction and Background}

The aim of this research is to develop modelling techniques to simulate and predict the behaviour of thinwalled composite structures in which the fibres are bonded by a matrix that is orders of magnitude softer than standard resin. These soft matrix structures are of interest to build compliant hinges in deployable structures. Experimental observation shows that one-ply textile composites with matrix made of materials such as silicone can be folded to a much higher curvature than those made with traditional epoxy. For example, Figure 1 shows a sheet of single-ply triaxial weave of carbon fibre tows with standard epoxy resin everywhere apart from a $3 \mathrm{~mm}$ wide strip that has been impregnated with silicone. A sharp crease coaxial with the centreline of the silicone strip can be introduced in the sheet and no apparent damage occurs even when the sheet is folded back to back.

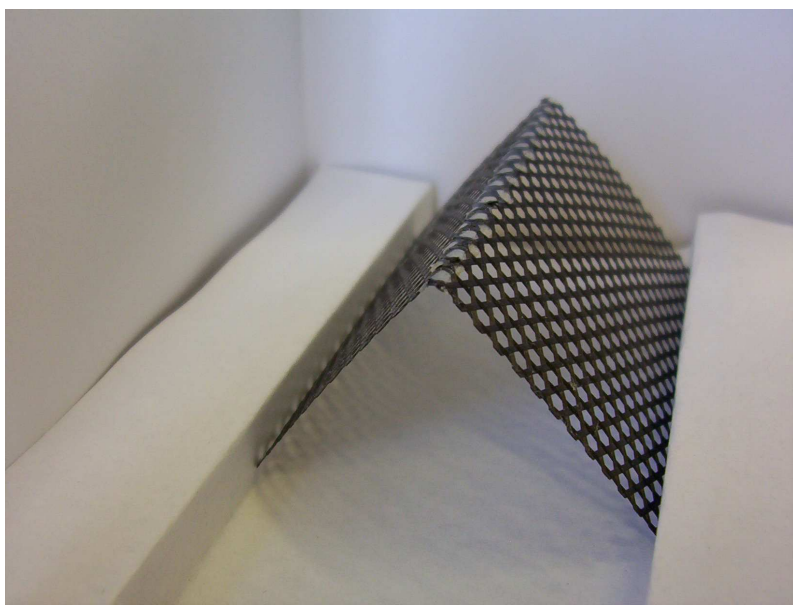

Figure 1. Flat sheet of single-ply triaxial weave composite with a $90^{\circ}$ localized elastic fold.

This research argues that the reason is the capability of the fibres to move within such a compliant matrix. While conventional resin is stiff enough to force the fibres to maintain their relative position, a

\footnotetext{
*Graduate Student, Graduate Aeronautical Laboratories, 1200 E. California Blvd. MC 301-46. f1@caltech.edu

$\dagger$ Professor of Aeronautics and Civil Engineering, Graduate Aerospace Laboratories, 1200 E. California Blvd. MC 301-46. AIAA Fellow. sergiop@caltech.edu
} 
much softer matrix will allow the fibres to move with respect to each other. In particular, this allows the compressed fibres to form a series of microbuckles.

Fibre microbuckling is a well known failure mode for composite materials. Rosen (1965) studied the problem using Timoshenko and Gere's (1936) solution for the buckling of a beam on an elastic foundation. Assuming a sinusoidal shape for the buckled fibres, the method compares the work done by the external forces with the strain energy in the system; the strain energy of the matrix is included in the analysis. Rosen considers two different cases, corresponding to different deformed configurations. In the first one the matrix deforms in extension, while in the second one it shears. The second configuration requires a lower critical load, and it is therefore considered the preferred one.

Micro-buckling has also been identified as a stress relieving mechanism in elastic memory composites by Murphey et al. (2001) and Campbell et al. (2004). This effect has been observed experimentally (Francis et al., 2006; Francis, 2008) and used in the design of space structures (Schultz, 2007; Campbell et al., 2005). The analyses carried out so far follow Rosen's work, but have included a number of modifications to the strain energy of the matrix. Figure 2 shows a sketch of the deformation of the fibres. When folding starts, the stiffness of the fibres is uniform throughout the thickness of the material, and the neutral axis lies in the middle plane. As the fibres on the compression side reach their critical buckling load, their stiffness is reduced, according to the post-buckling mechanics of the fibres. This produces a bilinear constitutive model, shifting the neutral axis from the middle surface to the tensile side of the laminate. This effect reduces the maximum strain in the fibres, allowing for a smaller elastic radius of curvature of the laminate to be achieved.

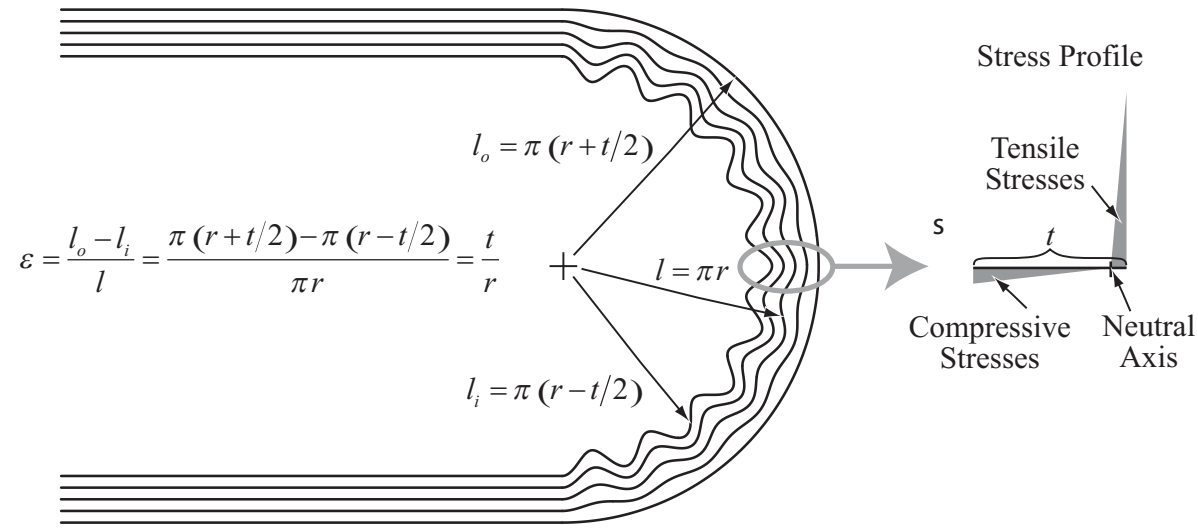

Figure 2. Fibre microbuckling and stress profile in a heavily bent laminate, taken from Murphey et al. (2001).

The goal of this paper is to develop an analytical model for the folding of composite materials with unidirectional fibres embedded in a soft matrix. The first step is to characterize the buckling behaviour of the fibres; their critical load and the wave length of their deformed shape are of particular interest. It will be shown that there are significant differences between fibre buckling when the composite material is subjected to uniform compression or to a bending moment. Once the microbuckling of the fibres has been analysed, the model is extended to include its effect on the macroscopic folding of the composite, to allow the determination of the moment-curvature relationship and of the strain in the fibres, in order to predict the curvature at which the composite material fails. Hull and Clyne (1996) state that the radius of carbon fibres typically used in composite materials is of the order of $4 \mu \mathrm{m}$ and that their breaking curvature is typically around $2.7 \mathrm{~mm}^{-1}$ (corresponding to a surface strain of approximately $1 \%$ ). Using this value as an upper limit for fibre curvature, it is shown that fibre microbuckling allows the composite material to remain undamaged at much higher curvatures.

Before starting this analytical work, a series of detailed finite element simulations of the micro-mechanics of the composite material were carried out. The finite element model consists of a repetitive unit cell of a single tow of uniaxial fibres embedded in a soft matrix. These simulations provide a more complete understanding of the buckling process, as well as the kinematics in the post buckling regime. Additionally, the results will be used to test the predictions from the analytical model. 


\section{Finite Element Model}

The composite material has been modeled as an elastic continuum using the finite element package ABAQUS/Standard. The fibres form an hexagonal grid of cylindrical rods, and the distance between them is calculated as a function of the fibre volume ratio. The fibres have been modeled with 3D solid elements, instead of 1D beams, since the actual amount of material between the fibres affects the relative motion between them. Bonding between matrix and fibre is assumed to be perfect, and therefore both materials will share nodes on the contact surface.

In order to reduce the computational cost, only a repeating cell of the tow has been modeled. Defining $x$ as the direction of the fibres and $y$ as the perpendicular to the width of the tow, we will consider the strip obtained by cutting the tow with two planes parallel to the $x y$ plane, as seen in Figure 3. This strip has length $L$, width $W$ and height $H$, as defined in the figure.

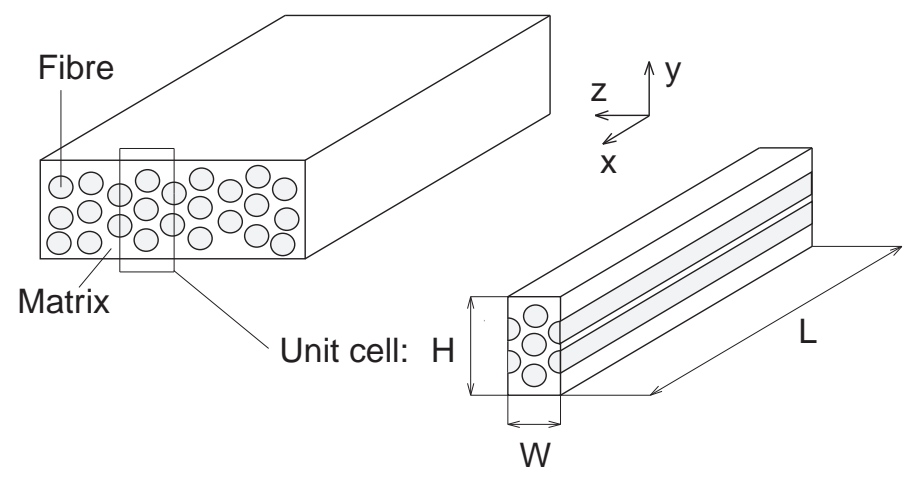

Figure 3. Unit cell

The boundary conditions on the faces of this unit cell must ensure that the deformation of the strip of material modeled is periodic in the $z$ direction. Since the meshing of the two faces is identical, periodic boundary conditions have been applied node by node, using the EQUATION command in ABAQUS. The displacement components in the three directions of every node are forced to be equal to those of the corresponding node in the opposite face.

The end cross sections of the unit cell correspond to parts of the tow where the fibres are embedded in standard epoxy resin, hence here the modulus of the matrix is much stiffer. It has been shown, by means of additional simulations that will not be reported here, that the relative position of the fibres remains practically unchanged in these end regions. Therefore, it can be assumed that the nodes in each end sections are fixed relative to one another and hence they can be connected to a single dummy node at the center of the section. In order to simulate the folding of the tow, equal and opposite rotations about $z$, of magnitude $\theta$, are applied to each dummy node. One node is constrained against translation in all directions, while the other one is free to translate along the $x$ axis. The faces perpendicular to the $y$ axis are left free. A geometrically non-linear simulation is carried out.

A sketch of the folding process is shown in Figure 4. The current radius of curvature, $R$, is calculated approximating the shape of the deformed strip with a circle, and it is used to calculate the current curvature, $\kappa$.

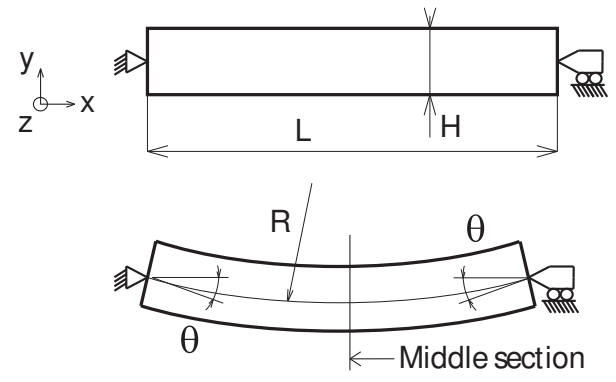

Figure 4. Schematic diagram of folding process 
In order to study the bending of the tow, the unit cell must extend through the whole thickness of the tow and for this reason a rectangular unit cell has been selected. Although the geometry of the fibres can be described by hexagonal or triangular unit cells, the variation of the deformation through the thickness of the tow dictates that a larger unit cell be used. There are two ways of fitting a rectangular, repeating unit cell onto a regular hexagonal grid, as seen in Figure 5. The main parameter to consider in this case is the orientation of the rectangle with respect to the hexagon generating the pattern. In the first of the two configurations shown in Figure 5 the periodic side of the unit cell is parallel to one of the edges of the hexagon. In the second one they are perpendicular. Each configuration is obtained by rotating the other one by $30^{\circ}$, and the unit cells are invariant to rotations of $60^{\circ}$.

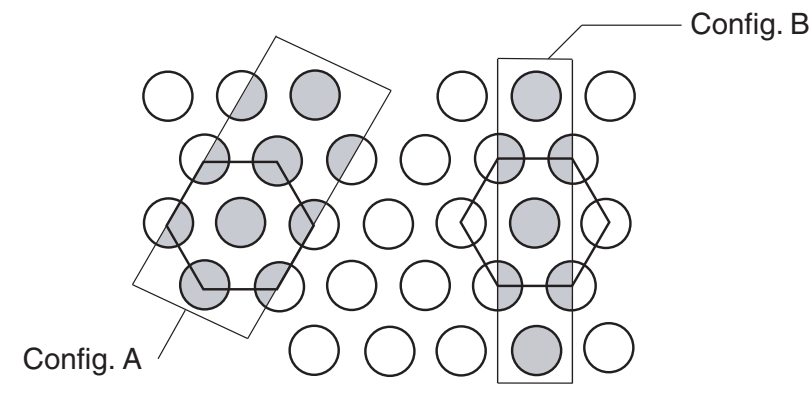

Figure 5. Different rectangular unit cells for a given hexagonal grid

Micrographs of real materials, such as Figure 6, show that in reality there is considerable irregularity in the lattice structure and hence there is not a single unit cell that correctly describes the physical situation. Therefore, both of these orientations have been used during the simulations, and the differences discussed.

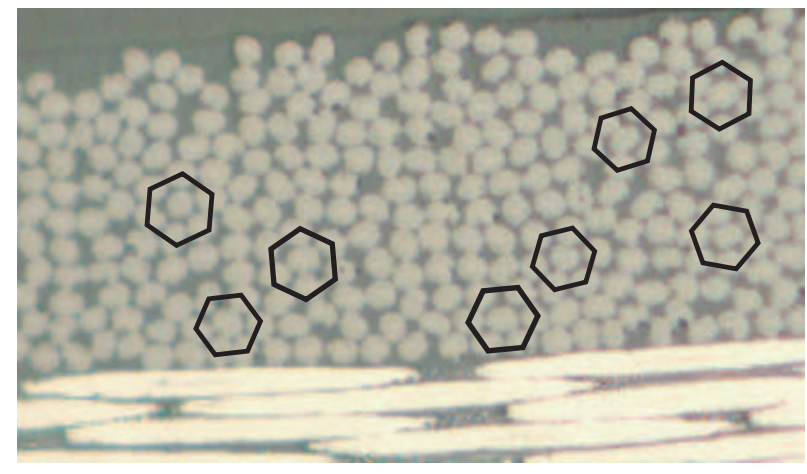

Figure 6. Micrograph showing hexagonal arrangements of fibres

Since all cross sections perpendicular to the $x$-axis are identical, the mesh will be the same for all of them. It is a two dimensional mesh, created by iterative bisection of an initial, very coarse mesh. The nodes in the surface of the fibres lie in circular manifolds. This way, the coordinates of the newly created nodes are adjusted to satisfy the equation of the circle. Figure 7 shows the initial mesh, an intermediate step, and the result mesh. 

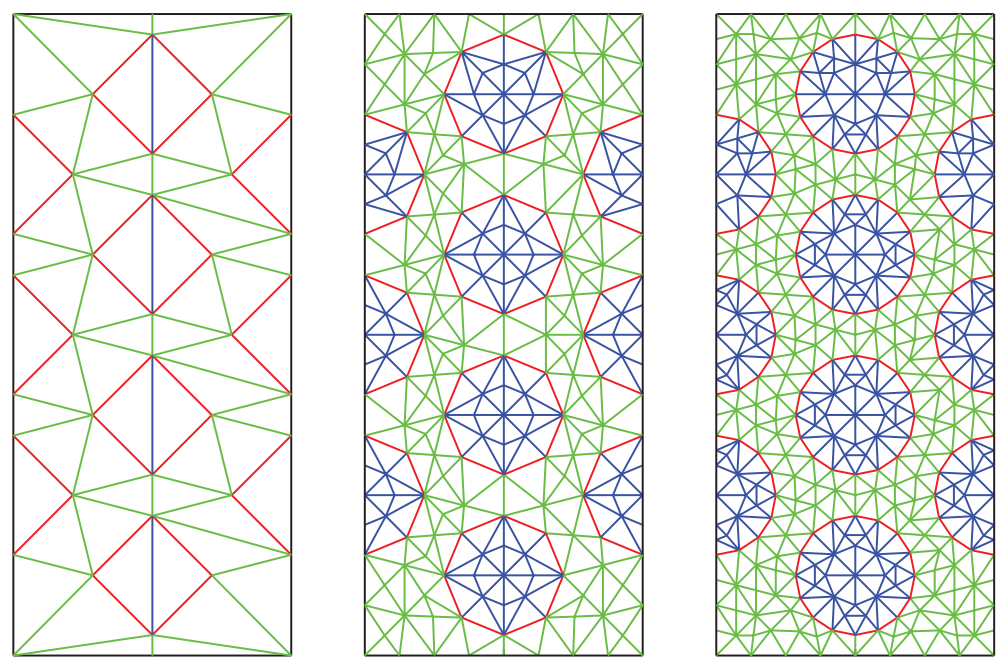

Figure 7. Initial, intermediate and final mesh for a section

Once the mesh for the cross-section has been completed, it is repeated through the strip, at equally spaced intervals. This creates the final tridimensional mesh, whose elements are triangular prisms. Triangular, 6node linear prism elements elements (C3D6) are used. Both the fibres and the matrix have been modeled as isotropic elastic materials; their properties are presented in Table 1.

\begin{tabular}{lc}
\hline \hline Material Properties & \\
\hline \hline fibre & \\
Young's Modulus & $200 \mathrm{GPa}$ \\
Poisson's ratio & 0.3 \\
\hline \hline Matrix (hard) & \\
Young's Modulus & $4.5 \mathrm{GPa}$ \\
Poisson's ratio & 0.35 \\
\hline \hline Matrix (soft) & \\
Young's Modulus & $1.5 \mathrm{MPa}$ \\
Poisson's ratio & 0.45 \\
\hline \hline Geometric Properties & \\
\hline \hline Radius of fibre & $4 \mu \mathrm{m}$ \\
Volume ratio & 0.5 \\
\hline \hline
\end{tabular}

Table 1. Material and geometric properties

For each type of unit cell configuration, results will be shown for one particular number of fibres. The example chosen for Configuration A contains four full fibres, while the example for Configuration B includes only three. These numbers have been picked so the overall height $H$ of the unit cells are similar. The dimensions of the cells are shown in Table 2 .

\section{Results of Finite Element Simulations}

This section shows the results of a series of simulations that have been carried out. All analyses were geometrically nonlinear analyses, and were conducted until ABAQUS aborted due to lack of convergence. The main cause is believed to be the excessive distortion in the elements of the matrix as they try to accommodate the movement of the fibres. The command STABILIZE has been used to introduce some numerical damping. The default value, $2 \cdot 10^{-4}$, has been used. 


\begin{tabular}{lc}
\hline \hline \multicolumn{2}{l}{ Geometric Properties } \\
\hline \hline Configuration A \\
$\mathrm{L}$ & $2 \mathrm{~mm}$ \\
$\mathrm{H}$ & $0.0431 \mathrm{~mm}$ \\
$\mathrm{~W}$ & $0.0187 \mathrm{~mm}$ \\
\hline \hline Configuration B \\
$\mathrm{L}$ & $2 \mathrm{~mm}$ \\
$\mathrm{H}$ & $0.0560 \mathrm{~mm}$ \\
$\mathrm{~W}$ & $0.0108 \mathrm{~mm}$ \\
\hline \hline
\end{tabular}

Table 2. Geometric properties

Figure 8 shows a general view of the result of a simulation, where both the applied curvature and the effects of fibre buckling can be seen. A series of cross sections show more clearly the displacement of the fibres within the matrix, as a result of micro buckling. It can clearly be seen how the elements corresponding to the matrix are heavily distorted, especially those between two fibres in different states of buckling.

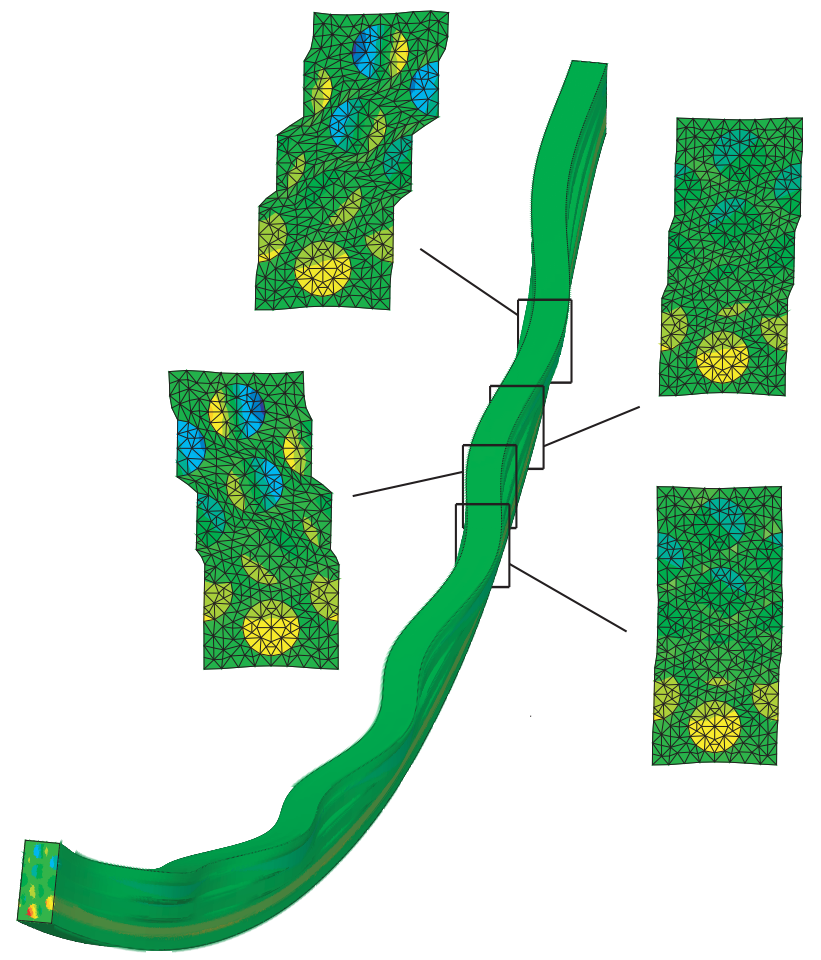

Figure 8. Perspective view of deformed configuration

The shape of the buckled fibres, as well as their wavelength, can be seen more easily in the top view in Figure 9. The deformation in the $z$ direction has been magnified by a factor of two, in order to make the buckling effects more clear. This amplification will be used in every top view, unless stated otherwise. Eleven approximately equal half sines of wavelength $\lambda$ can be identified. Also, some overall curvature can be observed. Unless stated otherwise, all of the plots that will be presented are taken at this section. 


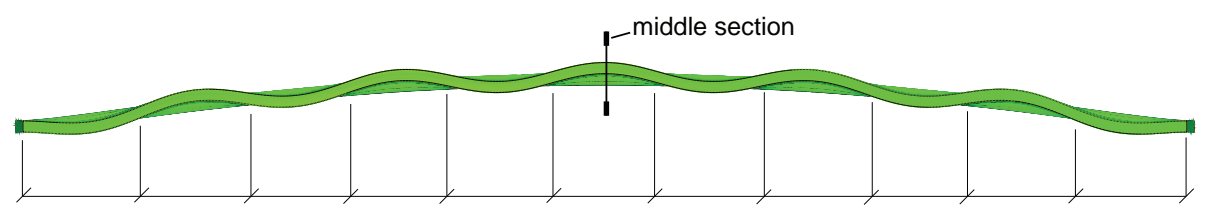

Figure 9. Top view of deformed configuration

Figure 10 shows the the longitudinal stress $\sigma_{11}$ at two different points of the simulation. The first plot corresponds to an approximate curvature $\kappa$ of $0.14 \mathrm{~mm}^{-1}$. In this case it is possible to see how the stress in the top fibre does not vary uniformly with the coordinate $y$. This implies that there is already some bending in the fibre. This moment corresponds to the beginning of the buckling. The second plot shows the moment in which the simulation stops, at $\kappa=0.29 \mathrm{~mm}^{-1}$. Here the top two fibres are completely buckled, with stress going from negative to positive. The bottom two fibres, although in compression, show some variation in the stress due to bending.
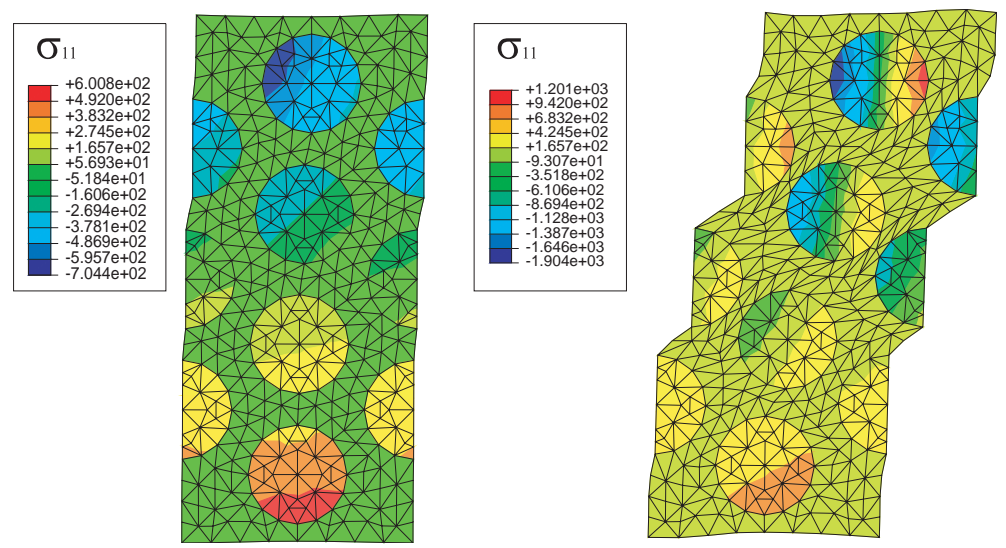

Figure 10. Longitudinal stresses at two different points in the simulation

Figure 11 shows the two relevant shear strain terms in the matrix. The first plot shows $\gamma_{13}$, which is the term considered by Rosen and all of the researchers that followed on. This plot has not been taken in the same section as in the other ones, but at a section in which the buckling deflection is zero. The second plot shows $\gamma_{23}$. This term can be neglected when the buckling is produced by pure compression, since all the fibres buckle approximately together. However, in the case of bending, buckling starts in the compression side, and it is not homogeneous. The fibres have different deflection, and this produces strain in the $y z$ plane.
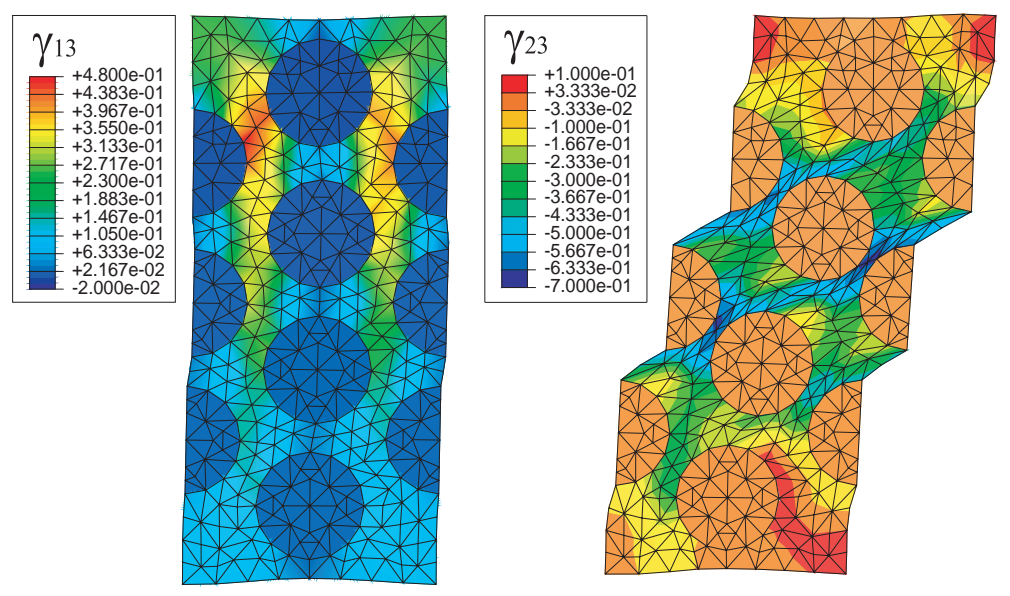

Figure 11. Shear strains 
Each plot shows the maximum values of the corresponding shear strain. This means that both terms are out of phase. This can be seen more clearly in the horizontal section shown in Figure 12. The maxima of $\gamma_{13}$ coincide with the zeros of the deformed shape. In the case of $\gamma_{23}$, the maximum strain occurs together with the maximum buckling deflection. This effect will be discussed in more detail in Section IV.

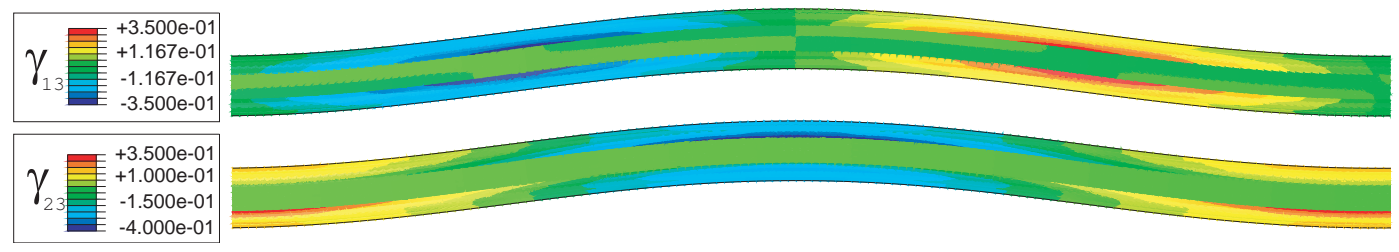

Figure 12. Shear strains

One of the main parameters to check is the strain in the fibres. The first plot in Figure 13 shows the principal strains in a section. The high values in the matrix are due to the shear strain. The values in the fibres are much lower.

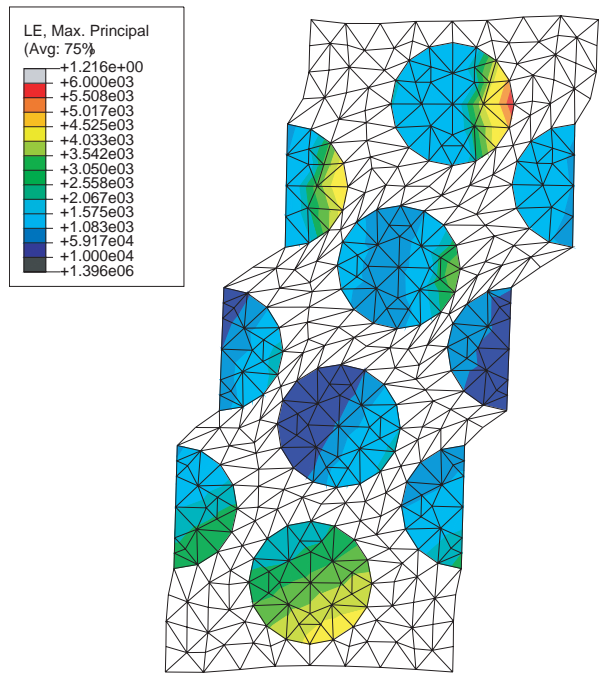

Figure 13. Principal strain in fibres

The results obtained for configuration B are similar to those presented before. Figure 14 shows the longitudinal stress, and the same features appearing in the previous case can be observed here. Figure 15 shows the two relevant shear terms. Again, the behaviour follows closely that seen in the previous case. 


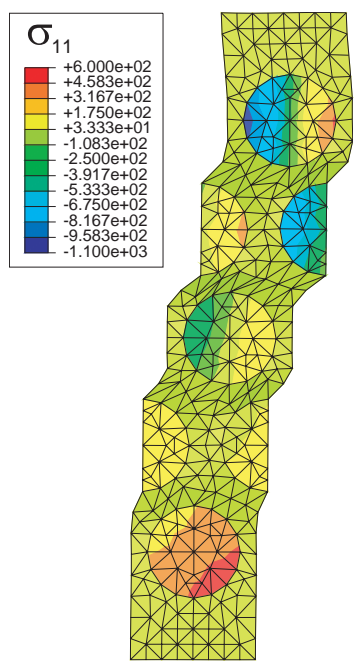

Figure 14. Normal stress in configuration B
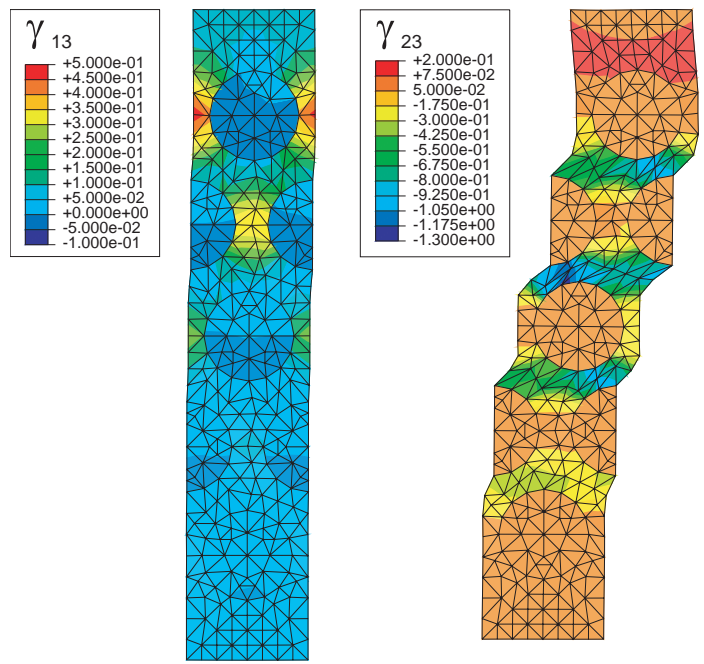

Figure 15. Shear strains in configuration B

\section{Buckling Analytical Study}

A simple analytical model has been used to study the buckling of the fibres. It will be used to predict the two main parameters of the process, the critical load and the wavelength of the deformed fibre. It also explains some of the features observed in the simulation results.

The model will treat the fibres as beams with square cross section. This is done in order to simplify the kinematics, particularly the deformation in the matrix. The fibres will have a side of length $h_{f}$, such that both cross sections have the same area:

$$
h_{f}=\sqrt{\pi} r_{f}
$$

With this value it is possible to calculate the new value of the second moment of area $I_{f}$. It should be noted, however, that the difference with the case of a circular section is of only $4.7 \%$.

The unit cell considered for this model will be the one shown in Figure 16. Every fibre and the matrix around it form a square of size $h$, given by $h_{f}$ and the volume ratio $V_{f}$ : 


$$
h=\frac{h_{f}}{\sqrt{V_{f}}}
$$

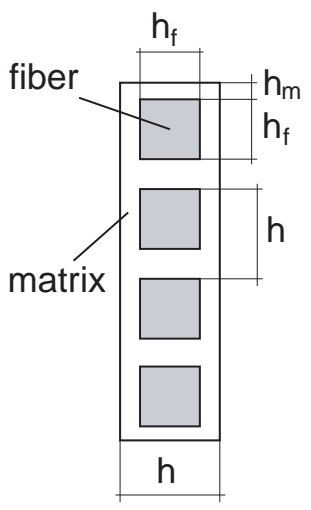

Figure 16. Model for analytical study

As stated before, the main difference with buckling due to uniform compression is the fact that in this case the fibres buckle progressively. This model will assume that all fibres buckle at the same critical stress $\sigma_{c r}$. It is also assumed that a certain number of fibres start buckling at the same time, and that more fibres continue to buckle as the compression stress reaches $\sigma_{c r}$.

It will be assumed first that only one fibre buckles, with deflection $\delta$. The deformed configuration is as shown in Figure 17. The value of $\delta$ will vary along the material, with equation:

$$
\delta=a(\xi) \sin \frac{m \pi x}{L}
$$

where $a$ is the amplitude, and $m$ is the number of half sines per length $L$.

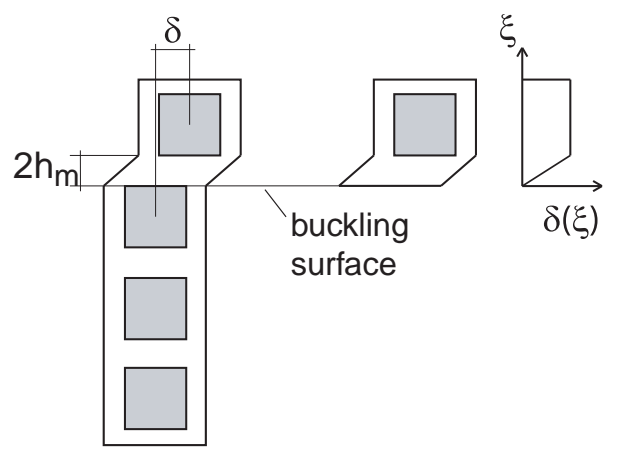

Figure 17. Buckling kinematics in analytical model - One buckled fibre

The deflection is then dependent not only on $x$, but also on $\xi$. This coordinate, parallel to $y$, measures the distance to the buckling surface. This is defined as the surface in which the value of the buckling deflection stops being zero. If only one fibre buckles, this surface coincides with the top of the second fibre. It is assumed that $a$ varies linearly until the next fibre, and is constant afterwards. For every given cross section (i.e., for a fixed value of $x$ ) the deflection $\delta$ is proportional to $a$, and therefore shows the same behavior. This can be seen in Figure 17.

These kinematics create two different regions in the matrix, as seen in Figure 18. The value of the amplitude of the deflection, $a$, is different in the two regions, and so is the value of the shearing terms. 


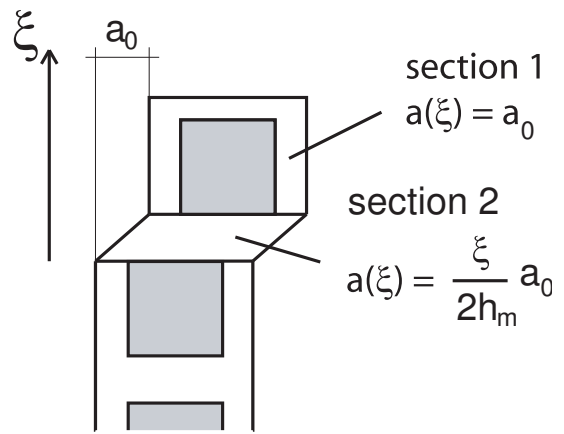

Figure 18. Variation of buckling deflection amplitude

The buckling analysis can then be performed considering only the buckled fibre and the sheared matrix. The goal is to obtain expressions for the critical load and the wavelength of the buckled fibre. Both are obtained through the same energy calculation, equating the work done by external loads with the strain energy in the material. The procedure presented here follows the method described by Rosen (1965).

The external energy is equal to:

$$
\Delta T=P_{c r} \frac{\pi^{2} m^{2} a_{0}^{2}}{4 L}
$$

where $P_{c r}$ is the critical buckling load.

The strain energy of a buckled fibre is:

$$
\Delta U_{\text {fibre }}=\frac{\pi^{4} E_{f} I_{f} m^{4} a_{0}^{2}}{4 L^{3}}
$$

where $E_{f}$ and $I_{f}$ are the Young's modulus and second moment of inertia of the fibres.

The strain energy of the matrix has two components, due to $\gamma_{x z}$ and $\gamma_{y z}$. The values are different in each of the sections shown in Figure 18.

In the first section, the only shear strain present is $\gamma_{x z}$. The expression is the same considered by Rosen:

$$
\gamma_{x z, 1}=\left(1+\frac{h_{f}}{2 h_{m}}\right) \frac{\partial \delta}{\partial x}=\left(1+\frac{h_{f}}{2 h_{m}}\right) \frac{a_{0} m \pi}{L} \cos \frac{m \pi x}{L}
$$

The factor $\left(1+\frac{h_{f}}{2 h_{m}}\right)$ takes into account the effect of the finite radius of the fibres. It was introduced by Rosen. The corresponding strain energy is then:

$$
\Delta U_{x z, 1}=\frac{1}{4} G_{m} \frac{a_{0}^{2} m^{2} \pi^{2}}{L}\left(1+\frac{h_{f}}{2 h_{m}}\right)^{2} h_{m}\left(h+2 h_{f}\right)
$$

where $G_{m}$ is the shear modulus of the matrix, and the term $h_{m}\left(h+2 h_{f}\right)$ gives the cross sectional area of the fraction of the matrix considered.

In the second section, both $\gamma_{x z}$ and $\gamma_{y z}$ are present. The value of the first one is:

$$
\gamma_{x z, 2}=\frac{a(\xi) m \pi}{L} \cos \frac{m \pi x}{L}
$$

The corresponding strain energy is equal to:

$$
\Delta U_{x z, 2}=\frac{1}{4} G_{m} \frac{a_{0}^{2} m^{2} \pi^{2}}{L} \frac{2 h_{m}}{3} h
$$

Finally, this section also presents $\gamma_{y z}$ shearing. It is constant, with value:

$$
\gamma_{y z}=\frac{\delta}{2 h_{m}}=\frac{a(\xi) \sin \frac{m \pi x}{L}}{2 h_{m}}
$$


The strain energy is

$$
\Delta U_{y z}=\frac{1}{24} G_{m} L \frac{a_{0}^{2}}{h_{m}} h
$$

The external energy should then be equal to the sum of strain energies:

$$
\Delta T=\Delta U_{f i b r e}+\Delta U_{x z, 1}+\Delta U_{x z, 2}+\Delta U_{y z}
$$

Substituting the previous values and simplifying, this expression turns into:

$$
P_{c r} \frac{\pi^{2} m^{2}}{L}=\frac{\pi^{4} E_{f} I_{f} m^{4}}{L^{3}}+G_{m} \frac{m^{2} \pi^{2}}{L} h_{m}\left(\left(1+\frac{h_{f}}{2 h_{m}}\right)^{2}\left(h+2 h_{f}\right)+\frac{2 h}{3}\right)+\frac{1}{6} G_{m} L \frac{h}{h_{m}}
$$

which does not depend on $a_{0}$.

It is possible now to calculate the critical load, which will be equal to:

$$
P_{c r}=\frac{\pi^{2} E_{f} I_{f}}{\lambda^{2}}+G_{m} h_{m}\left(\left(1+\frac{h_{f}}{2 h_{m}}\right)^{2}\left(h+2 h_{f}\right)+\frac{2 h}{3}\right)+\frac{1}{6} G_{m} \frac{h \lambda^{2}}{\pi^{2} h_{m}}
$$

where $\lambda=\frac{L}{m}$ is the wave length.

The first term in the equation corresponds to the contribution of the fibre, and is equal to the Euler critical load for buckling. Its contribution to the critical load decreases as the wavelength increases, as expected. The second term, corresponding to the shear term $\gamma_{x z}$, is constant. The additional shear term, $\gamma_{y z}$, increases with the wavelength. This effect is the one providing a minimum.

The contribution of the three terms, as well as the total load, can be seen in Figure 19.

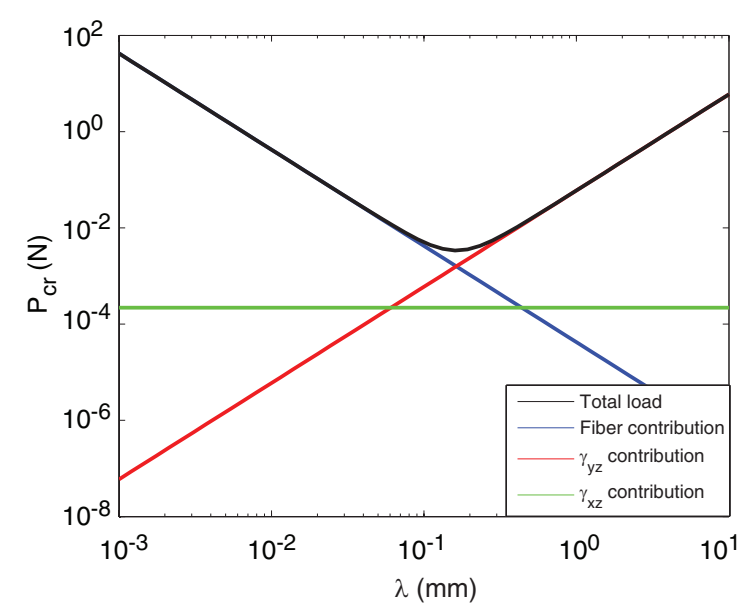

Figure 19. Critical load of one fibre as a function of wavelength

An expression for the minimum can be obtained analytically by minimizing $P_{c r}$ with respect to $\lambda$, and it is equal to:

$$
\lambda_{c r}=\pi\left(\frac{2 E_{f} I_{f} h_{m}}{3 G_{m} L h}\right)^{\frac{1}{4}}
$$

The homogenized critical stress $\tilde{\sigma}_{c r}$ is obtained dividing the critical load by the area of the unit cell. It is assumed that this value remains constant during the post buckle regimen.

Figure 20 shows the kinematics in the case of more than one fibre buckling.

The deflection $\delta$ varies linearly with respect to the fibres. In the case of $n$ fibres buckling, the amplitudes will have the expression

$$
a_{i}=a_{0} \frac{i}{n} \quad i=1, \ldots, n
$$


The energy terms are then:

$$
\begin{aligned}
\Delta T & =P_{c r} \frac{\pi^{2} m^{2}}{4 L} \sum a_{i}^{2} \\
\Delta U_{f i b r e} & =\frac{\pi^{4} E_{f} I_{f} m^{4}}{4 L^{3}} \sum a_{i}^{2} \\
\Delta U_{x z, 1} & =\frac{1}{4} G_{m} \frac{m^{2} \pi^{2}}{L}\left(1+\frac{h_{f}}{2 h_{m}}\right)^{2} h_{m}\left(h+2 h_{f} \sum a_{i}^{2}\right) \\
\Delta U_{x z, 2} & =\frac{1}{4} G_{m} \frac{m^{2} \pi^{2}}{L} \frac{2 h_{m} h}{3} n a_{0}^{2} \\
\Delta U_{y z} & =\frac{1}{24} G_{m} L \frac{a_{0}^{2} h}{n h_{m}}
\end{aligned}
$$

where it has been assumed that the critical load is the same for all buckled fibres. The first three terms increase as $n$ increases. The last term, on the other hand, decreases as $n$ increases. The reason is that for a given maximum buckling deflection, more fibres imply a lower $\gamma_{y z}$. Since the strain is squared in the energy calculation, the decrease in strain is more significant than the increase in material being sheared.

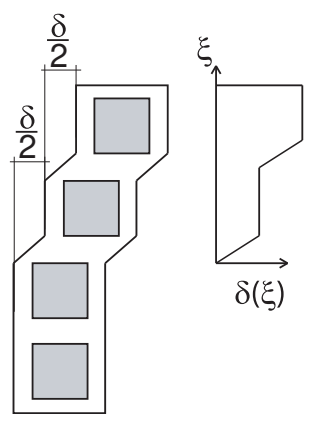

Figure 20. Buckling kinematics in analytical model - Several buckled fibres

Figure 21 shows the critical load for different cases, and how the critical load approaches the asymptote corresponding to the Euler buckling critical load as $n$ increases.

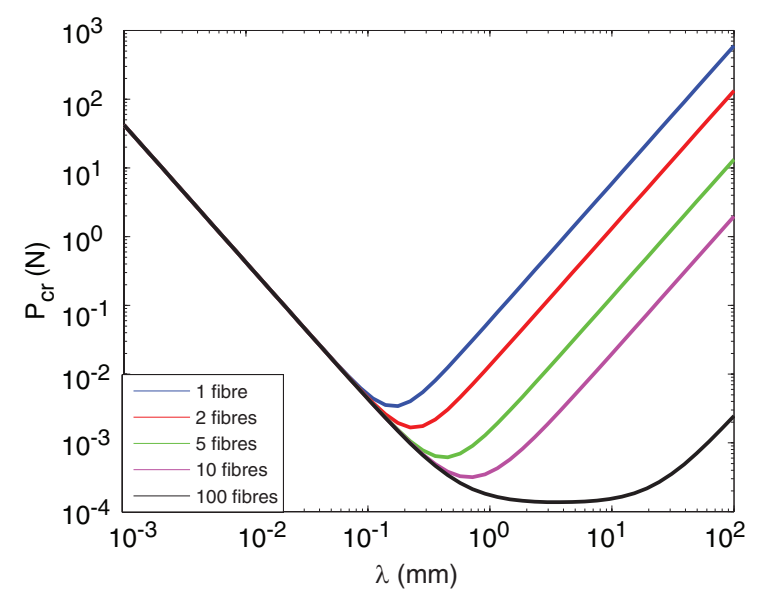

Figure 21. Critical load of a fibre as a function of wavelength for different numbers of buckled fibres

For large values of $n$ it is possible to use the approximation:

$$
\sum a_{i}^{2}=a_{0}^{2} \frac{n^{2}+(n-1)^{2}+\ldots+1}{n^{2}} \sim a_{0}^{2} \frac{n}{3}
$$


In this case the wavelength can be approximated by:

$$
\lambda_{c r} \sim \frac{\pi}{\sqrt{n}}\left(\frac{2 E_{f} I_{f} h_{m}}{G m L h}\right)^{\frac{1}{4}}
$$

A method to predict the number of fibres buckling in each case has not been developed yet. Observation of numerical simulations shows that the number of buckled fibres increases with the thickness, but no clear relation has been observed.

\section{Homogenized Folding Process}

The results of the buckling analysis allow the study of the folding of the material. As stated before, the material behaves linearly until buckling occurs. Then the neutral surface starts shifting towards the compression side, due to the lower stiffness of the buckled fibres. The analysis presented here is based on an homogenization process, such as that shown in Figure 22. Two different constitutive laws will be considered, one for the material before buckling, and a different one for the post-buckled regime. Both compression and tension will be present in the elastic regime. Once the homogenization is completed, the buckling of new fibres, as well as the shifting of the neutral surface, will be treated as a continuous effect. The distance from the geometric centroid to the neutral surface, which indicates the progress of the buckling, will be labeled $\alpha \frac{H}{2}$.

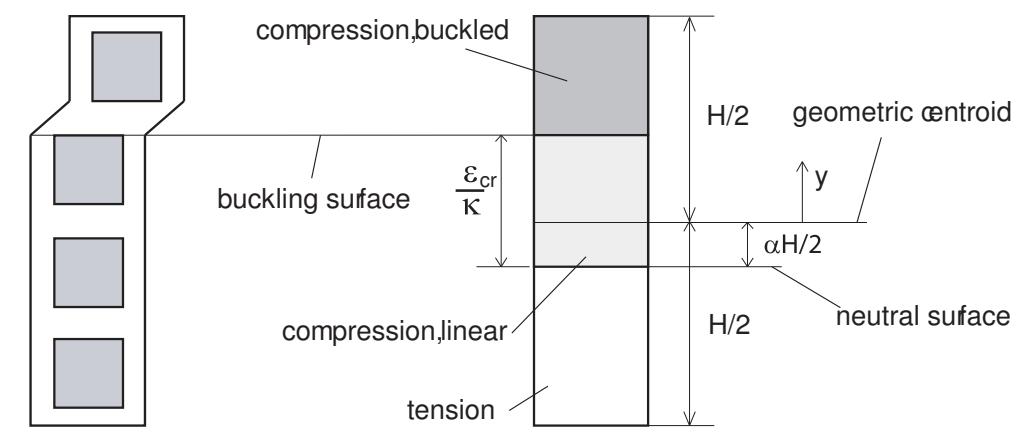

Figure 22. Cross section showing homogenization of linear and buckled regions

In the case of non buckled material, the elastic modulus will be given by the rule of mixtures:

$$
\tilde{E}=E_{f} V_{f}+E_{m} V_{m}
$$

where $\tilde{E}$ is the homogenized elastic modulus.

In the post-buckled region, it is assumed that the material carries constant stress, equal to $\tilde{\sigma}_{c r}$. The resultant material behaviour, shown in Figure 23, is similar to that of a elastic - perfectly plastic material.

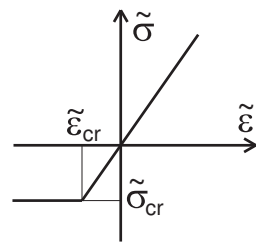

Figure 23. Homogenized constitutive law

The folding behaviour of such a material is well known. It has also been studied in the context of buckled composite materials (Campbell et al., 2004). When the folding starts, the material behaves linearly until buckling occurs. The critical curvature will be related with the critical homogenized strain as: 


$$
\kappa_{c r}=\frac{2 \epsilon_{c r}}{H}
$$

Up to this point, the moment per unit width will be equal to:

$$
M=\tilde{E} I \kappa=\frac{1}{12} \tilde{E} H^{3} \kappa
$$

where $I$ is the second moment of inertia per unit width of the section, treated as a single material.

Once the fibres start buckling, the neutral surface starts shifting towards the compression side. It is possible to calculate its position using equilibrium. The stress field in the linear field is equal to:

$$
\tilde{\sigma}=\tilde{E} \tilde{\epsilon}=\tilde{E}\left(\kappa y-\kappa \alpha \frac{H}{2}-\tilde{\epsilon}_{c r}\right)
$$

As stated before, the stress in the buckled region is constant and equal to $\tilde{\sigma_{c r}}$. The total stress, obtained by integrating the field over the section, must be zero:

$$
\int_{\alpha \frac{H}{2}}^{\frac{H}{2}} \tilde{E}\left(\kappa y-\kappa \alpha \frac{H}{2}-\tilde{\epsilon}_{c r}\right) d y+\int_{-\frac{H}{2}}^{\alpha \frac{H}{2}} \tilde{\sigma}_{c r} d y=0
$$

This can be reduced to:

$$
\alpha=1-\sqrt{\frac{8 \tilde{\epsilon}_{c r}}{\kappa H}}
$$

Once the position of the neutral surface has been calculated, it is possible to calculate the moment. It is equal to the integral of the stress times the distance to the surface:

$$
M=\int_{\alpha \frac{H}{2}}^{\frac{H}{2}} \tilde{E} \kappa\left(y-\alpha \frac{H}{2}-\frac{\tilde{\epsilon}_{c r}}{\kappa}\right)^{2} d y+\int_{-\frac{H}{2}}^{\alpha \frac{H}{2}} \tilde{\sigma}_{c r}\left(y-\alpha \frac{H}{2}-\frac{\tilde{\epsilon}_{c r}}{\kappa}\right) d y
$$

Using $\tilde{\sigma}_{c r}=\tilde{E} \tilde{\epsilon}_{c r}$, this expression turns into:

$$
\begin{aligned}
M & \left.=\tilde{E} \kappa\left(\frac{y^{3}}{3}-y^{2}\left(\alpha \frac{H}{2}+\frac{\tilde{\epsilon}_{c r}}{\kappa}\right)+y\left(\alpha \frac{H}{2}+\frac{\tilde{\epsilon}_{c r}}{\kappa}\right)^{2}\right)\right]_{\alpha \frac{H}{2}}^{\frac{H}{2}} \\
& \left.-\tilde{E} \tilde{\epsilon}_{c r}\left(\frac{y^{2}}{2}-y\left(\alpha \frac{H}{2}+\frac{\tilde{\epsilon}_{c r}}{\kappa}\right)\right)\right]_{-\frac{H}{2}}^{\alpha \frac{H}{2}}
\end{aligned}
$$

The expression can be finally reduced to:

$$
M=\tilde{E} \frac{H^{3} \kappa}{12}\left(\frac{1}{2}(1-\alpha)^{3}-\frac{3}{4 \eta}\left(1-6 \alpha+\alpha^{2}\right)+\frac{3}{\eta^{2}}\right)
$$

where $\eta$ is equal to the maximum strain obtained for the same curvature, normalized by the critical buckling strain:

$$
\eta=\frac{\kappa H}{2 \tilde{\epsilon}_{c r}}
$$

\section{Fibre Failure Analysis}

The curvature that produces failure in a fibre can also be calculated once the position of the neutral surface is known. It will be assumed that the fibres buckled when the strain reaches a critical level. The maximum strain for both the tension and compression regions will be calculated.

The strain in the tensioned fibres is equal to the curvature times the maximum distance to the neutral axis. Using Equation 29, the maximum strain is:

$$
\epsilon_{\text {tension }}=\sqrt{2 \tilde{\epsilon}_{c r} H \kappa}-\tilde{\epsilon}_{c r}
$$


For the compression side, it is possible to calculate the maximum strain in the fibres due to bending. The curvature in a fibre of sinusoidal shape is equal to:

$$
\kappa_{f}=\frac{y^{\prime \prime}}{\left(1+\left(y^{\prime}\right)^{2}\right)^{\frac{3}{2}}}=\frac{-\left(\frac{\pi}{\lambda}\right)^{2} h \sin \frac{\pi x}{\lambda}}{\left(1+\left(\frac{\pi}{\lambda} h \cos \frac{\pi x}{\lambda}\right)^{2}\right)^{\frac{3}{2}}}
$$

where $a$ is the amplitude of the sine.

The maximum curvature takes place at the peak of the sine, and it is equal to:

$$
\kappa_{f, \max }=\frac{\pi^{2} a}{\lambda^{2}}
$$

The maximum strain in the fibre due to the buckling will be then equal to:

$$
\epsilon_{\text {compression }}=r_{f} \kappa_{f, \max }=\frac{r_{f} \pi^{2} a}{\lambda^{2}}
$$

In order to compute it, it is necessary to calculate first the wave length and amplitude of the buckled fibre. The wave length can be calculated using the fact that, in the post buckled region, the homogenized strain is due to the difference between the original length of the fibre and the buckled wavelength:

$$
\tilde{\epsilon}=\frac{\lambda-\lambda_{0}}{\lambda_{0}}
$$

where $\lambda$ is the current wave length and $\lambda_{0}$ the initial wave length calculated in the previous section. This value will be taken as the length of the fibre corresponding to a half sine. This way, the wave length in the post buckled fibre changes, but every half sine corresponds to the same original length.

The homogenized strain at the end of the buckled region is equal to:

$$
\tilde{\epsilon}=\kappa\left(H-\sqrt{2 \tilde{\epsilon}_{c r} H \kappa}\right)+\tilde{\epsilon}_{c r}
$$

and so the wavelength is equal to:

$$
\lambda=\lambda_{0}+\lambda_{0}\left(\kappa\left(H-\frac{H}{2} \sqrt{2 \tilde{\epsilon}_{c r} H \kappa}\right)+\tilde{\epsilon}_{c r}\right)
$$

The amplitude of the sine can be calculated equating the deformed and undeformed lengths:

$$
\lambda_{0}=\int_{0}^{\lambda_{0}} \sqrt{1+\frac{\pi^{2} a^{2}}{\lambda^{2}} \cos x \pi \lambda}
$$

This equation can be solved numerically. This will provide both $\lambda$ and $a$, and so the maximum strain in the buckled fibres can be calculated.

\section{Results and Discussion}

\section{A. Wavelength}

Two numerical models, one for each configuration, have been used to predict the value of the wave-length for pieces of material of approximately the same thickness. The simulation done with configuration A gave a wavelength of $0.18 \mathrm{~mm}$. In the case of case $\mathrm{B}$, the result was $0.28 \mathrm{~mm}$. The difference is probably due to the fact that there is more matrix between fibres in the $y$ for configuration $\mathrm{B}$. This implies that the matrix deforms with lower energy (due to lower $\gamma_{y z}$ ), and so the wave-length is higher.

The result of the analytical model would depend on the numbers of buckled fibres considered. The values obtained for one, two or three fibres are $0.1758 \mathrm{~mm}, 0.2223 \mathrm{~mm}$ and $0.2812 \mathrm{~mm}$, respectively. This values are all close to those obtained with the simulation. The main requirement is now the development of a process to determine the number of fibres buckled. 


\section{B. Moment - Curvature}

Figure 24 shows the moment - curvature obtained through simulations and the analytical work. The simulations considered configuration A (4 fibres, $0.0431 \mathrm{~mm}$ thickness) and configuration B (6 fibres, 0.1120 $\mathrm{mm}$ thickness). Cases with the same thickness have been studied with the analytical model. It captures the overall behaviour, but there are significant differences. The case with higher thickness seems to provide a better agreement. This would agree with the fact that the homogenization process should work better for a larger thickness, in which the continuum approximation is better justified.

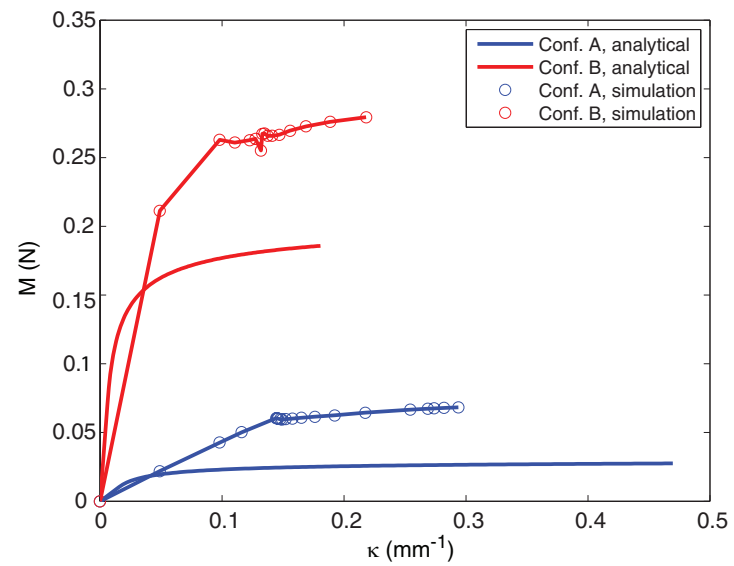

Figure 24. Moment per unit length vs. curvature

\section{Failure Strain}

Figure 25 shows the results of the failure analysis. It has been assumed that there are 4, 10 and 20 fibres through the thickness, and that half the fibres buckle. It can be seen how the strain in the tensioned fibres are greatly reduced. The strain in the buckled fibres is also reduced, specially when compared with the reference case, in which no buckling is allowed. The reduction is even larger if more fibres are considered.

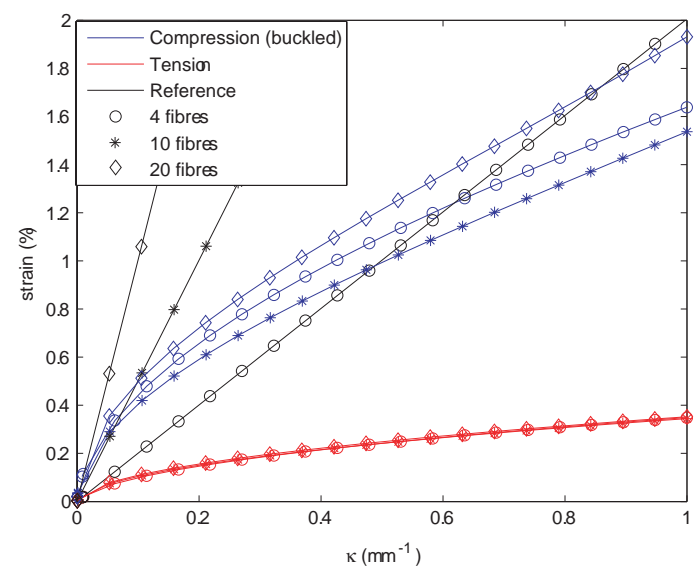

Figure 25. Strain at the fibres

\section{Conclusions and Future Work}

Detailed micromechanical finite element simulations of composite materials with soft materials have been presented. The simulations allow the study of micro-buckling under bending, including the kinematics in the fibres, as well as the strains in the matrix. This model has shown substantial differences with the models 
used before, which considered buckling under uniform compression.

These simulations have led to the creation of a simple analytical model that captures most of the features observed in the simulation. This model, although simple, allows a quite accurate estimation of the buckling wavelength. It also provides the moment-curvature relationship, but this last result presents significant differences with the simulations. Finally, the model is also capable of predicting the maximum strain in the fibres for a given curvature.

Future work should first focus in refining the analytical model. The goal will be to obtain an homogenized model of the buckling process. It should also treat the fibres in a more realistic way. This model might predict the material properties more accurately.

More simulations should also be produced. It would be interesting to check different cases, varying the number of fibres, length of the piece considered, and possibly the fibre pattern. Also, a solution to the high distortion in the mesh could produce simulations that achieve a higher curvature.

It will also be interesting to provide experimental results of the process study. This would allow contrasting the validity of both the simulations and the analytical model. It would also be useful to investigate other effects, impossible to capture in the model presented here. In particular, the effects of an irregular matrix on the wavelength, as opposed to the regular patterns considered here. Experimental evidence could explain if the wavelength is given by the part of the material buckling first, or if it is the result of some averaging process.

Once the model is completed and verified, it will be useful in designing deployable structures that take advantage of the properties of the materials studied here. In particular, it could be used to design structures with localized folds and increased packaging ratio.

\section{References}

Campbell, D., Lake, M.S., and Mallick, K. (2004) A Study of the Bending Mechanics of Elastic Memory Composites, 45th AIAA/ASME/ASCE/AHS/ASC Structures, Structural Dynamics, and Materials Conference, Palm Springs, CA, April 19-22, AIAA-2004-1636.

Campbell, D., Lake, M.S., Scherbarth, M.R., Nelson, E., and Six, R. W. (2005), Elastic Memory Composite Material: An Enabling Technology for Future Furlable Space Structures, 46th AIAA/ASME/ASCE/AHS/ASC

Structures, Structural Dynamics, and Materials Conference, Austing, TX, April 18-21, AIAA 2005-2362

Francis, W. H., Lake, M.S., and Steven Mayes, J. (2006) A Review of Classical fiber Microbuckling Analytical Solutions for use with Elastic Memory Composites, 47th AIAA/ASME/ASCE/AHS/ASC Structures, Structural Dynamics, and Materials Conference, Newport, RI, May 1-3, AIAA-2006-1764.

Francis, W. H. (2008) Mechanics of Post-Microbuckled Compliant-Matrix Composites, Master of Science Thesis, Colorado State University.

Hull, D., and Clyne, T.W. (1996) An Introduction to Composite Materials, Second Edition, Cambridge University Press.

Murphey, T.W., Meink, T., and Mikulas, M.M. (2001) Some micromechanics considerations of the folding of rigidizable composite materials, 42nd AIAA/ASME/ASCE/AHS/ASC Structures, Structural Dynamics, and Materials Conference, AIAA-2001-1418.

Rosen, B.W. (1965) Fiber Composite Materials, American Society for Metals, Metals Park, Ohio, 1965, Ch. 3.

Schultz, M.R., Francis, W.H., Campbell, D., and Lake, M.S. (2007) Analysis Techniques for ShapeMemory Composite Structures, 48th AIAA/ASME/ASCE/AHS/ASC Structures, Structural Dynamics, and Materials Conference, Honolulu, HI, April 23-26, AIAA 2007-2401.

Timoshenko, S. P., and Gere, J. M. (1961) Theory of Stability, McGraw-Hill Book Company. 